\title{
Hardware Implementation of Image Recognition System Based on Morphological Associative Memories and Discrete Wavelet Transform
}

\author{
Enrique Guzmán ${ }^{1}$, Selene Alvarado ${ }^{1}$, Oleksiy Pogrebnyak ${ }^{2}$, \\ Luis Pastor Sánchez Fernández ${ }^{2}$, and Cornelio Yañez ${ }^{2}$ \\ ${ }^{1}$ Universidad Tecnológica de la Mixteca \\ eguzman@mixteco.utm.mx. \\ ${ }^{2}$ Centro de Investigación en Computación del Instituto Politécnico Nacional \\ (olek, lsanchez, cyanez) @pollux.cic.ipn.mx
}

\begin{abstract}
The implementation of a specific image recognition technique for an artificial vision system is presented. The proposed algorithm involves two steps. First, smaller images are obtained using Discrete Wavelet Transform (DWT) after four stages of decomposition and taking only the approximations. This way the volume of information to process is reduced considerably and the system memory requirements are reduced as well. Another purpose of DWT is to filter noise that could be induced in the images. Second, the Morphological Associative Memories (MAM) are used to recognize landmarks. The proposed algorithm provides flexibility, possibility to parallelize algorithms and high overall performance of hardware implemented image retrieval system. The resulted hardware implementation has low memory requirements, needs in limited arithmetical precision and reduced number of simple operations. These benefits are guaranteed due to the simplicity of MAM learning/restoration process that uses simple morphological operations, dilation and erosion, in other words, MAM calculate maximums or minimums of sums. These features turn out the artificial vision system to be robust and optimal for the use in realtime autonomous systems. The proposed image recognition system has, among others, the following useful features: robustness to the noise induced in the patter to process, high processing speed, and it can be easily adapted to diverse operation circumstances.
\end{abstract}

Keywords: Artificial Vision, Image Recognition, Morphological Associative Memories, Discrete Wavelet Transform, Hardware Implementation.

\section{Introduction}

Currently the artificial vision of autonomous systems has a grown interest in the scientific community and in the potential industry applications as well. The main problem of the artificial vision is the recognition of contained in images physical elements and determining their identity and position. [1]. Diverse techniques have been used for the image pattern recognition in artificial vision, the most known are listed below. 
Lin-Cheng Wang et al. [1] described a modular neural network classifier for the automatic object recognition based on forward-looking infrared imagery. The classifier is formed by several independently trained neuronal networks; each neuronal network makes a decision based on local features extracted from a specific part of the image to recognize, then individual results of each network are combined to determine the final decision.

Dong-Gyu Sim, Oh-Kyu Kwon, and Rae-Hong Park [2] proposed algorithms for object recognition based on modification of Hausdorff distance algorithm. These modifications uses M-estimation and least trimmed square measures, which demonstrated are more efficient than the conventional Hausdorff distance measures.

A new approach for object recognitions based on coarse-and-fine matching and a multilayer Hopfield neural network was presented by Susan S. Young and Peter D. Scott in [3]. The network is formed by several cascade single layer Hopfield networks, each network codifies object features at different resolutions, with bidirectional interconnections linking adjacent layers.

Susan S. Young et al. presented a method to detect and classify objects using a multi resolution neuronal network [4]. The objects identification is based on minimizing an energy function. This energy function is evaluated by means of a procedure of concurrent equalization implemented with a multilayer Hopfield neuronal network.

An algorithm based on wavelets analysis for identification and separation of packages in the recycling process is proposed by J.M. Barcala et al. in [5]. Quaternions are constructed with obtained wavelet coefficients that allow to realize the identification of the packages on-line. The objective of this system is its use in recycling plants replacing manual process.

Foltyniewicz, R.[6] presented a new method for automatic face recognition and verification. His approach is based on a two stage process. At the first step a wavelet decomposition technique or morphological nonlinear filtering is used to enhance intrinsic features of a face, reduce the influence of rotation in depth, changes in facial expression, glasses and lighting conditions. Preprocessed images contain all the essential information for the discrimination between different faces and are next a subject for learning by a modified high order neural network which has rapid learning convergence, very good generalization properties and a small number of adjustable weights.

Considering an image as a pattern, we propose the hardware implementation of image recognition for an artificial vision system based on Morphological Associative Memories and Discrete Wavelet Transform. The designed system outperforms the traditionally artificial vision techniques in the robustness to noise, learning ability, high speed of both learning and recognition processes and overall efficiency of the image patterns recognition.

\section{Morphological Associative Memories}

The modern era of associative memories began in 1982 when Hopfield described the Hopfield Associative Memory in [7]. In 1998, a novel work appears in this area when 
Ritter, Sussner and Diaz de León created Morphological Associative Memories (MAM). The MAM base their operation on the morphological operations, dilation and erosion. In other words, they use the maximums or minimums of sums [8]. This feature distinguishes them from the Hopfield memories, which use sums of products.

The input patterns and output patters of the Mg generic associative memory are represented by $\mathbf{x}=\left[x_{i}\right]_{n}$ and $\mathbf{y}=\left[y_{i}\right]_{m}, \quad$ respectively. Let $\left\{\left(\mathbf{x}^{1}, \mathbf{y}^{1}\right),\left(\mathbf{x}^{2}, \mathbf{y}^{2}\right), \ldots,\left(\mathbf{x}^{k}, \mathbf{y}^{k}\right)\right\}$ be $k$ vector pairs defined as the fundamental set of associations [10]. The fundamental set of associations is described as

$$
\left\{\left(\mathbf{x}^{\mu}, \mathbf{y}^{\mu}\right) \mid \mu=1,2, \ldots, k\right\}
$$

The Mg associative memory is represented by a matrix and is generated from the fundamental set of associations. Once the fundamental set is delineated, one can use the necessary operations for the learning and recovery processes of MAM. These operations are the maximum product and minimum product and they use the maximum $\bigvee$ and minimum $\wedge$ operators [9], [10], [11].

According to the operation mode, the associative memories can be classified in two groups: Morphological Auto-associative Memories (MAAM) and Morphological Hetero-Associative Memories (MHM). Morphological Heteroassociative Memories are of particular interest for the development of this work.

\subsection{Morphological Heteroassociative Memories}

A MAM is hetero-associative if $\exists \mu \in\{1,2, \ldots, k\}$ such that $\mathbf{x}^{\mu} \neq \mathbf{y}^{\mu}$. There are two types of MHM: $\max$, symbolized by $\mathbf{M}$, and $\min$, symbolized by $\mathbf{W}$.

The $\mathbf{W}$ memories are those that use the maximum product and the minimum operator in their learning phase and the maximum product in their recovery phase; the $\mathbf{M}$ memories are those that use the minimum product and the maximum operator in their learning phase and the minimum product in their recovery phase.

\section{Learning phase:}

1. For each of the $k$ element of the fundamental set of associations $\left(\mathbf{x}^{\mu}, \mathbf{y}^{\mu}\right)$, the matrices $\mathbf{y}^{\mu} \nabla\left(-\mathbf{x}^{\mu}\right)^{t}$ are calculated for a $\mathbf{W}$ memory, or the matrices $\mathbf{y}^{\mu} \Delta\left(-\mathbf{x}^{\mu}\right)^{t}$ are calculated for a $\mathbf{M}$ memory. The morphological heteroassociative memory is created.

2. The $\mathbf{W}$ memory is obtained applying the minimum operator $\wedge$ to the resulting matrices of step $1 . \mathbf{W}$ is given by

$$
\begin{gathered}
\mathbf{W}=\bigwedge_{\mu=1}^{k}\left[\mathbf{y}^{\mu} \nabla\left(-\mathbf{x}^{\mu}\right)^{t}\right]=\left[w_{i j}\right]_{m \times n} \\
w_{i j}=\bigwedge_{\mu=1}^{k}\left(y_{i}^{\mu}-x_{j}^{\mu}\right)
\end{gathered}
$$

The memory $\mathbf{M}$ is obtained applying the maximum operator $V$ to the resulting matrices of step 1. $\mathbf{M}$ is given by 


$$
\begin{gathered}
\mathbf{M}=\underset{\mu=1}{\vee}\left[\mathbf{y}^{\mu} \boldsymbol{\Delta}\left(-\mathbf{x}^{\mu}\right)^{t}\right]=\left[m_{i j}\right]_{m \times n} \\
m_{i j}=\bigvee_{\mu=1}^{k}\left(y_{i}^{\mu}-x_{j}^{\mu}\right)
\end{gathered}
$$

\section{Recovery phase:}

The recovery phase consists of presenting an input pattern to the memory generated at the learning phase. As an answer, the memory generates the output pattern associated to the presented pattern.

When $\mathbf{W}$ memory is used, the maximum product $\mathbf{W} \nabla \mathbf{x}^{\omega}$ is calculated,

$$
\begin{gathered}
\mathbf{y}=\mathbf{W} \nabla \mathbf{x}^{\omega} \\
y_{i}=\bigvee_{j=1}^{n}\left(w_{i j}+x_{j}^{\omega}\right)
\end{gathered}
$$

When a $\mathbf{M}$ memory is used, the minimum product $\mathbf{M} \Delta \mathbf{x}^{\omega}$ is calculated,

$$
\begin{gathered}
\mathbf{y}=\mathbf{M} \Delta \mathbf{x}^{\omega} \\
y_{i}=\bigwedge_{j=1}^{n}\left(m_{i j}+x_{j}^{\omega}\right)
\end{gathered}
$$

where $\omega \in\{1,2, \ldots, k\}$, and a column vector $\mathbf{y}=\left[y_{i}\right]_{m}$ is obtained, which represents the output patterns associated with $\mathbf{x}^{\omega}$ input patterns.

Theorem 1 and Theorem 2 of [12] govern the conditions that must be satisfied respectively by MHM $\max$ and $\min$ to obtain a perfect recall to output patterns. On the other hand, Theorem 5 and Theorem 6 of [12] indicate the amount of noise that is permissible in the input patterns to obtain a perfect recall to output patterns.

\section{Hardware Implementation of Image Recognition System}

When an autonomous system uses an artificial vision technique, it is required that this system must be robust to certain factors such as the noise induced in the pattern to process. Besides, it can be able to develop high speeds of processing and be easily adapted to diverse circumstances of operation, among others.

In order to satisfy these requirements, we propose the hardware description of a specific application processor focused on patterns recognition in an artificial vision system. The proposed algorithm described in the processor uses MAM for the patterns recognition. Besides of before mentioned useful features that make MAM an attractive tool, MAM have demonstrated the excellent performance in recognizing and recovering patterns, even in presence of dilative, erosive or random noise [9], [10], [13]. Discrete Wavelet Transform (DWT) is a complement to the proposed algorithm. The use of DWT has two objectives. First, when one uses only the approximation sub-band of the four-scale DWT decomposition, the quantity of 
information to process is reduced and therefore the memory system requirements are reduced too. Second, DWT filters the noise that could be induced in the image at previous stages.

The proposed image recognition system combines the flexibility, the facility to describe parallel processes and a high performance that is granted by the implementation of an algorithm in hardware, and it inherits the MAM features such as low memory requirements, limited arithmetical precision, and small number of arithmetical operations.

The use of Electronic Design Automation (EDA) tools optimizes the system design time, increases the product quality and reduces the production costs. For the development of the proposed system, we have chosen the set of EDA tools formed by the software tools ISE (Integrated Software Environment) Foundation version 8.2 $\mathrm{i}$ from Xilinx company and the hardware descriptor language VHDL, and Xess XSV300 Board V1.0 is the hardware tool.

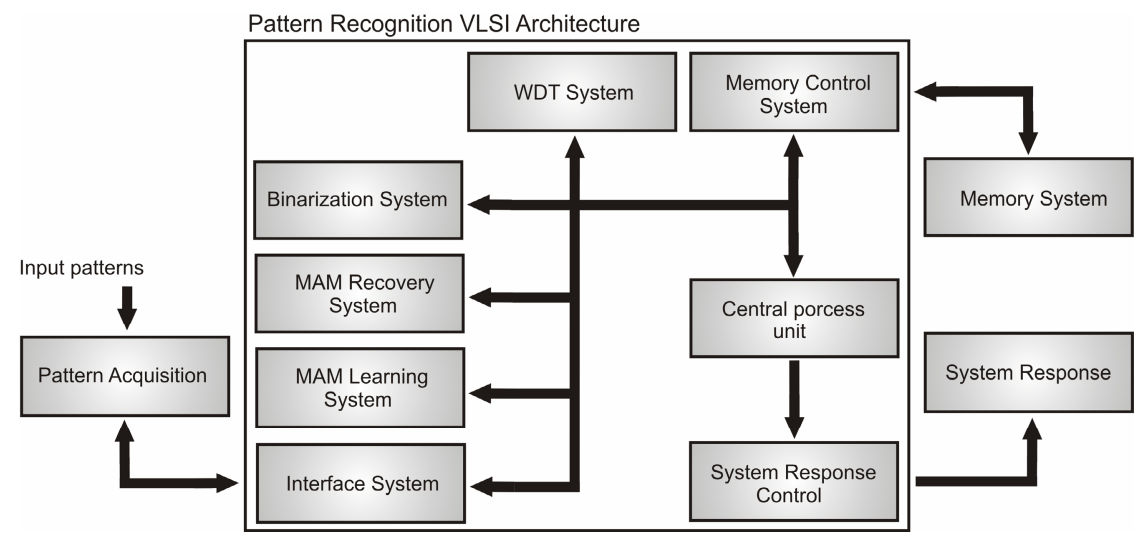

Fig. 1. Proposed system scheme

In order to describe the processor for patterns recognition, we choose the TopDown design methodology. It begins visualizing the design with a high level of abstraction. Then the design is partitioned to a further designs, each new design increases its level of detail according to the required one [14]. Based on the principles of Top-Down design methodology, our design is divided in modules shown in Fig. 1. Each of these modules is independent of another but all interact to each other constantly. The algorithm proposed for the design of the processor for patterns recognition consists of two phases, learning and recognition.

\subsection{Learning Phase}

At this phase, both MHM min and MHM max are created. These memories codify the information necessary to identify each one of the input patterns that are represented by images. Fig. 2 illustrates the learning algorithm. 


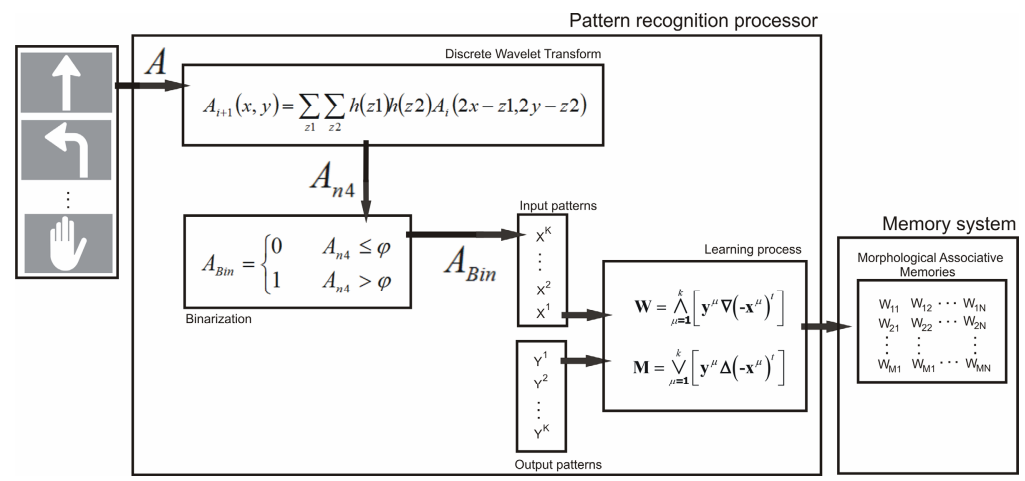

Fig. 2. Learning process

The learning algorithm can be summarized in the following stages.

i. Image acquisition. The implemented USB interface between the image recognition system and a personal computer allows emulating the image acquisition and visualizing the results.

Let $C I=\left\{A^{\alpha} \mid \alpha=1,2, \ldots h\right\}$ be the set of $h$ images to recognize; each image is represented by $A^{\alpha}=\left[a_{i j}\right]_{m x n}$, where $m$ represents the height of the image and $n$ the width of the image.

ii. The four-scale DWT decomposition of the image $A$ is computed [15]:

$$
A_{i+1}(x, y)=\sum_{z 1} \sum_{z 2} h(z 1) h(z 2) A_{i}(2 x-z 1,2 y-z 2)
$$

The following stage uses only the approximation sub-band, $A_{n 4}=\left\lfloor a_{i j}\right\rfloor_{u x v}$, where $u$ and $v$ define the size of the new image; detail sub-bands are discarded.

iii. The approximation sub-band of the four-scale DWT decomposition is converted in the binary image $A_{B i n}$ :

$$
A_{\text {Bin }}= \begin{cases}0 & A_{n 4} \leq \varphi \\ 1 & A_{n 4}>\varphi\end{cases}
$$

where $\varphi$ is a binary index.

iv. Conversion of $A_{B i n}$ to vector. This vector is an input pattern of the fundamental set of associations:

$$
\begin{gathered}
\mathbf{x}^{\mu}=A_{B i n}^{\mu} \mid \mu=1,2, \ldots, h \\
{\left[x_{l}\right]_{u v}=\left\lfloor a_{i j}\right\rfloor_{u x v}}
\end{gathered}
$$

v. A label is assigned to the vector $\mathbf{x}^{\mu}=A_{\text {Bin }}^{\mu}$, this label represents an output pattern $\mathbf{y}^{\mu} \mid \mu=1,2, \ldots, h$. The union of these patterns generates an element of the fundamental set of associations: $\left\{\left(\mathbf{x}^{\mu}, \mathbf{y}^{\mu}\right) \mid \mu=1,2, \ldots, h\right\}$. 
Theorem 1 and Corollary 1.1 in [12] govern the conditions that must be satisfied by output pattern for MHM max to obtain a perfect recall.

Theorem 2 and Corollary 2.1 in [12] govern the conditions that must be satisfied by output pattern for MHM min to obtain a perfect recall.

vi. The Morphological Associative Memories are obtained:

The MHM max are computed using both the minimum product $\Delta$ and the maximum operator $\mathrm{V}$

$$
\mathbf{M}=\underset{\mu=1}{\vee}\left[\mathbf{y}^{\mu} \Delta\left(-\mathbf{x}^{\mu}\right)^{t}\right]
$$

The MHM min are compute using both the maximum product $\nabla$ and the minimum operator $\wedge$

$$
\mathbf{W}=\wedge_{\mu=1}^{k}\left[\mathbf{y}^{\mu} \nabla\left(-\mathbf{x}^{\mu}\right)^{t}\right]
$$

vii. Stages i - vi are repeated $h$ times.

\subsection{Recognition Phase}

At this phase, the system is able to identify the images that represent the input patterns with the aid of the memory obtained in the learning phase. When an image is received, the system recovered the label (output pattern) that was associated to this image. Fig. 3 shows the recognition phase.

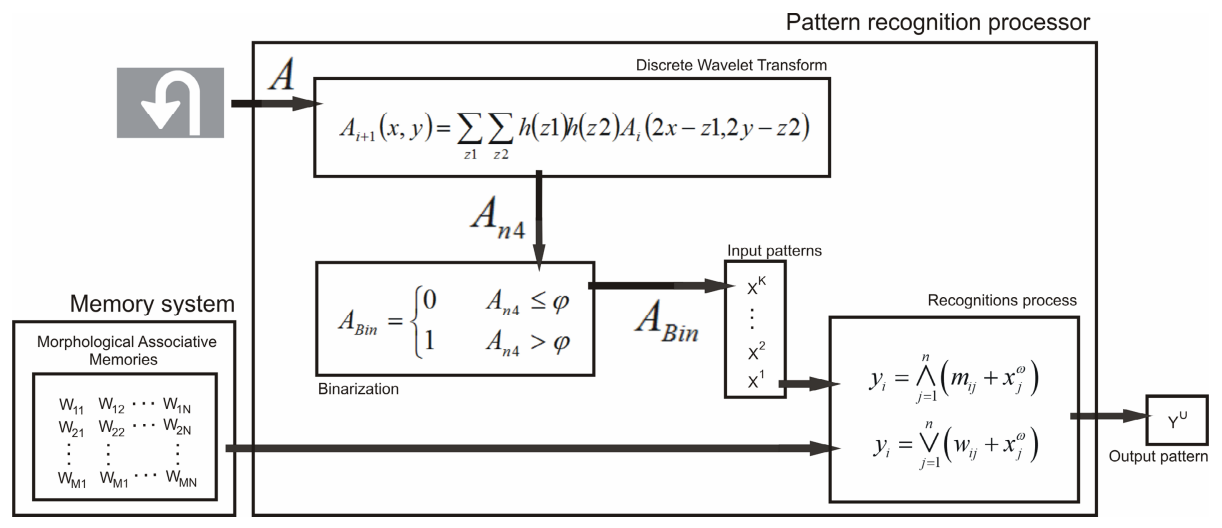

Fig. 3. Recognition process

The recognition algorithm can be summarized in the following stages.

i. Image to be identified is acquired.

ii. The four-scale DWT decomposition of the image is computed.

iii. The approximation sub-band of the four-scale WDT decomposition is converted into a binary image.

iv. The binary image is converted into vector.

v. With the help of the MAM obtained at learning phase the system is able to identify the received current image. The system generates the label corresponding to the identified image. 
If MHM max is used, the minimum product $\Delta$ is applied, $\mathbf{y}=\mathbf{M} \Delta \mathbf{x}^{\omega}$

If MHM $\min$ is used, the maximum product $\nabla$ is applied, $\mathbf{y}=\mathbf{W} \nabla \mathbf{x}^{\omega}$

vi. Finally, the generated label outputs to the response system for its following processing.

\section{Results}

In order to show the performance of the modeled image recognition system, we choose to apply it to 2 sets of images. The first image set is grayscale images of $720 x 480$ pixels shown in Fig. 4. Using the implemented USB interface between the image recognition system and a personal computer we can visualize the results of each one of the phases of the algorithm shown in Fig. 5. Table 1 shows a perfect recall for original images.

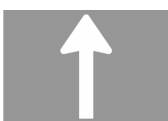

(a)

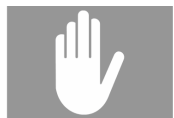

(b)

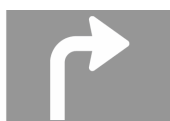

(c)

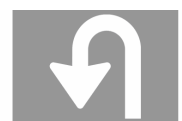

(d)

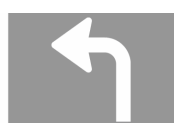

(e)

Fig. 4. Test images: a) go ahead, b) stop, c) right, d) return, e) left

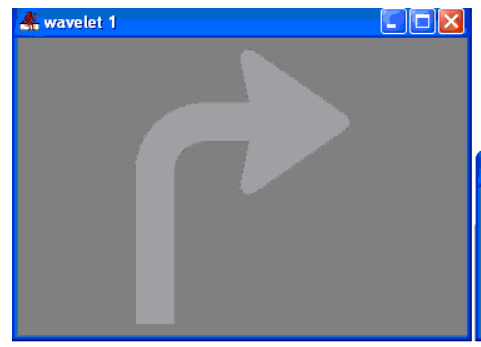

(a)

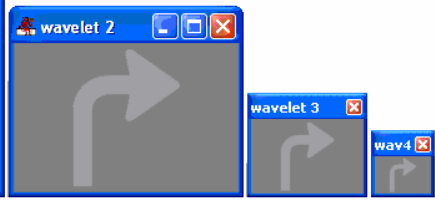

(b)

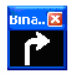

Fig. 5. Results of algorithm phases: a) approximation sub-bands of the WDT decomposition, b) binary process

Table 1. Processor for patterns recognition performance on original images

\begin{tabular}{ccc}
\hline & MHM $\min$ and MHM $\max$ performance (recognition percentage) \\
\cline { 2 - 3 } Image & MHM $\min$ & MHM $\max$ \\
\hline $\mathrm{a}$ & 100 & 100 \\
$\mathrm{~b}$ & 100 & 100 \\
$\mathrm{c}$ & 100 & 100 \\
$\mathrm{~d}$ & 100 & 100 \\
$\mathrm{e}$ & 100 & 100 \\
\hline
\end{tabular}

In order to estimate how the image recognition system performs in real conditions, the test images were corrupted with typically associated Gaussian noise and uniformly 
distributed noise. The images were corrupted with the 3 variants of each one of these noises: dilative, erosive and random. Fig. 6 and Tables 2, 3 and 4 compare the system performance using MHM $\max$ and MHM $\min$ on corrupted images with both Gaussian and uniform noise in 3 modalities: dilative, erosive and random, each with different noise percentages.

Table 2. Comparison of system performance using MHM max and MHM min on corrupted images with both dilative Gaussian and dilative uniform noise

\begin{tabular}{|c|c|c|c|c|c|c|c|c|c|c|c|c|}
\hline \multirow[b]{4}{*}{ Image } & \multicolumn{12}{|c|}{ MHM min and MHM max performance (recognition percentage) } \\
\hline & \multicolumn{6}{|c|}{ Dilative Gaussian noise } & \multicolumn{6}{|c|}{ Dilative Uniform noise } \\
\hline & \multicolumn{2}{|c|}{$10 \%$} & \multicolumn{2}{|c|}{$20 \%$} & \multicolumn{2}{|c|}{$30 \%$} & \multicolumn{2}{|c|}{$10 \%$} & \multicolumn{2}{|c|}{$20 \%$} & \multicolumn{2}{|c|}{$30 \%$} \\
\hline & $\begin{array}{c}\mathrm{MHM} \\
\min \end{array}$ & $\begin{array}{c}\text { MHM } \\
\max \end{array}$ & $\begin{array}{c}\mathrm{MHM} \\
\min \end{array}$ & $\begin{array}{c}\text { MHM } \\
\max \end{array}$ & $\begin{array}{c}\mathrm{MHM} \\
\min \end{array}$ & $\begin{array}{c}\text { MHM } \\
\max \end{array}$ & $\begin{array}{c}\mathrm{MHM} \\
\min \end{array}$ & $\begin{array}{c}\text { MHM } \\
\max \end{array}$ & $\begin{array}{c}\mathrm{MHM} \\
\min \end{array}$ & $\begin{array}{c}\text { MHM } \\
\max \end{array}$ & $\begin{array}{c}\mathrm{MHM} \\
\min \end{array}$ & $\begin{array}{c}\text { MHM } \\
\text { max }\end{array}$ \\
\hline a & 100 & 100 & 0 & 100 & 0 & 100 & 100 & 100 & 0 & 100 & 0 & 100 \\
\hline $\mathrm{b}$ & 100 & 100 & 0 & 100 & 0 & 100 & 0 & 100 & 0 & 100 & 0 & 100 \\
\hline $\mathrm{c}$ & 100 & 100 & 100 & 100 & 0 & 100 & 0 & 100 & 0 & 100 & 0 & 100 \\
\hline d & 100 & 100 & 100 & 100 & 0 & 100 & 100 & 100 & 100 & 100 & 100 & 100 \\
\hline e & 100 & 100 & 0 & 100 & 0 & 100 & 100 & 100 & 0 & 100 & 0 & 100 \\
\hline
\end{tabular}

Table 3. Comparison of system performance using MHM max and MHM min on corrupted images with both erosive Gaussian and erosive uniform noise

\begin{tabular}{|c|c|c|c|c|c|c|c|c|c|c|c|c|}
\hline \multirow[b]{4}{*}{ Image } & \multicolumn{12}{|c|}{ MHM min and MHM max performance (recognition percentage) } \\
\hline & \multicolumn{6}{|c|}{ Erosive Gaussian noise } & \multicolumn{6}{|c|}{ Erosive Uniform noise } \\
\hline & \multicolumn{2}{|c|}{$10 \%$} & \multicolumn{2}{|c|}{$20 \%$} & \multicolumn{2}{|c|}{$30 \%$} & \multicolumn{2}{|c|}{$10 \%$} & \multicolumn{2}{|c|}{$20 \%$} & \multicolumn{2}{|c|}{$30 \%$} \\
\hline & $\begin{array}{c}\text { MHM } \\
\text { min }\end{array}$ & $\begin{array}{c}\text { MHM } \\
\max \end{array}$ & $\begin{array}{c}\mathrm{MHM} \\
\min \end{array}$ & $\begin{array}{c}\text { MHM } \\
\max \end{array}$ & $\begin{array}{c}\text { MHM } \\
\text { min }\end{array}$ & $\begin{array}{c}\text { MHM } \\
\max \end{array}$ & $\begin{array}{c}\mathrm{MHM} \\
\text { min }\end{array}$ & $\begin{array}{c}\text { MHM } \\
\max \end{array}$ & $\begin{array}{c}\mathrm{MHM} \\
\text { min }\end{array}$ & $\begin{array}{c}\text { MHM } \\
\max \end{array}$ & $\begin{array}{c}\text { MHM } \\
\text { min }\end{array}$ & $\begin{array}{c}\text { MHM } \\
\max \end{array}$ \\
\hline $\mathrm{a}$ & 100 & 100 & 100 & 100 & 100 & 0 & 100 & 100 & 100 & 0 & 100 & 0 \\
\hline b & 100 & 100 & 100 & 100 & 100 & 100 & 100 & 100 & 100 & 100 & 100 & 100 \\
\hline $\mathrm{c}$ & 100 & 100 & 0 & 100 & 0 & 100 & 100 & 100 & 100 & 0 & 100 & 0 \\
\hline $\mathrm{d}$ & 100 & 100 & 100 & 100 & 100 & 0 & 100 & 100 & 100 & 0 & 100 & 0 \\
\hline $\mathrm{e}$ & 100 & 100 & 100 & 0 & 100 & 0 & 100 & 0 & 100 & 0 & 100 & 0 \\
\hline
\end{tabular}

Table 4. Comparison of system performance using MHM max and MHM min on corrupted images with both random Gaussian and random uniform noise

\begin{tabular}{|c|c|c|c|c|c|c|c|c|c|c|c|c|}
\hline \multirow[b]{4}{*}{ Image } & \multicolumn{12}{|c|}{ MHM min and MHM max performance (recognition percentage) } \\
\hline & \multicolumn{6}{|c|}{ Random Gaussian noise } & \multicolumn{6}{|c|}{ Random Uniform noise } \\
\hline & \multicolumn{2}{|c|}{$10 \%$} & \multicolumn{2}{|c|}{$20 \%$} & \multicolumn{2}{|c|}{$30 \%$} & \multicolumn{2}{|c|}{$10 \%$} & \multicolumn{2}{|c|}{$20 \%$} & \multicolumn{2}{|c|}{$30 \%$} \\
\hline & $\begin{array}{c}\mathrm{MHM} \\
\min \end{array}$ & $\begin{array}{c}\text { MHM } \\
\max \end{array}$ & $\begin{array}{c}\mathrm{MHM} \\
\min \end{array}$ & $\begin{array}{c}\text { MHM } \\
\max \end{array}$ & $\begin{array}{c}\mathrm{MHM} \\
\min \end{array}$ & $\begin{array}{c}\text { MHM } \\
\max \end{array}$ & $\begin{array}{c}\mathrm{MHM} \\
\min \end{array}$ & $\begin{array}{c}\text { MHM } \\
\max \end{array}$ & $\begin{array}{c}\mathrm{MHM} \\
\min \end{array}$ & $\begin{array}{c}\text { MHM } \\
\max \end{array}$ & $\begin{array}{c}\mathrm{MHM} \\
\min \end{array}$ & $\begin{array}{l}\text { MHM } \\
\max \end{array}$ \\
\hline a & 100 & 100 & 100 & 100 & 100 & 100 & 100 & 100 & 0 & 100 & 0 & 100 \\
\hline $\mathrm{b}$ & 100 & 100 & 100 & 100 & 0 & 100 & 100 & 100 & 0 & 100 & 0 & 100 \\
\hline $\mathrm{c}$ & 100 & 100 & 100 & 100 & 100 & 100 & 100 & 100 & 100 & 100 & 0 & 100 \\
\hline $\mathrm{d}$ & 100 & 100 & 100 & 100 & 100 & 100 & 100 & 100 & 100 & 100 & 0 & 100 \\
\hline $\mathrm{e}$ & 100 & 100 & 100 & 100 & 100 & 100 & 100 & 100 & 100 & 100 & 0 & 100 \\
\hline
\end{tabular}


(a)

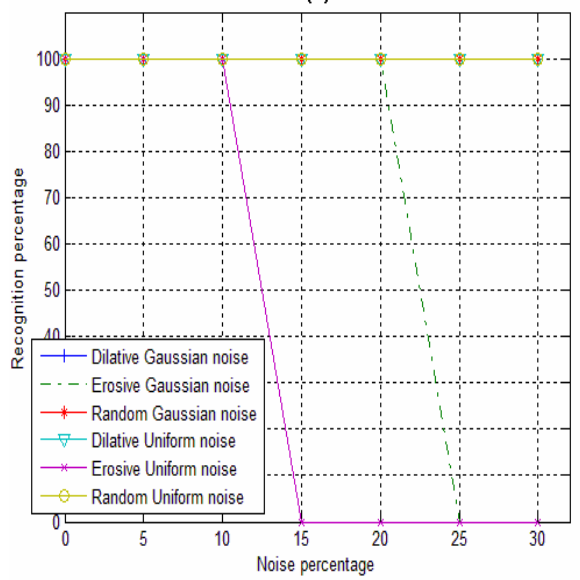

(b)

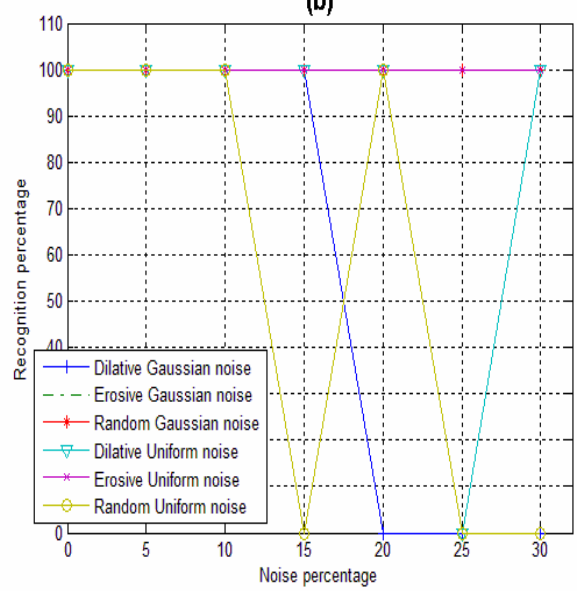

Fig. 6. MAM performance on corrupted "return” image, a) MHM max, b) MHM min

Observing the results, one can conclude that the image recognition system based on MHM max has a perfect recall when the image is corrupted by random noise, being this type of noise the one that commonly is added to images at previous stages before the recognition in the artificial vision system.

Second image set are grayscale images of 128x128 pixels shown in Fig 7.

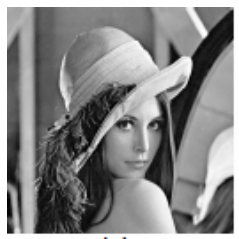

(a)

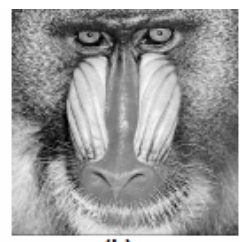

(b)

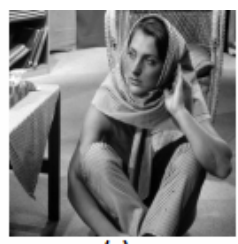

(c)

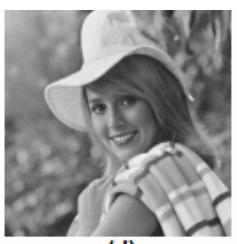

(d)

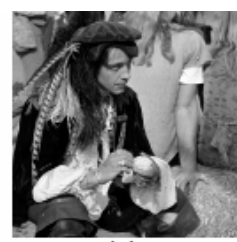

(e)

Fig. 7. Second test on images. a) Lena, b) Baboon, c) Barbara, d) Elaine, e) Man.

With aid of implemented USB interface between the image recognition system and the personal computer, we can visualize the results of each one of the phases of the algorithm shown in Fig. 8. Fig. 9 shows the results of the binarization of the test images implemented in the image recognition system. Table 5 shows a perfect recall to original images.

In order to estimate the behavior of the image recognition system in real conditions, the test images were corrupted with both dilative and erosive Gaussian noise. Table 6 compares the system performance using MHM min on corrupted images with different noise percentage.

The binarization process affects the performance of the image recognition system. The influence of the binarization can be estimated analyzing the results shown in 


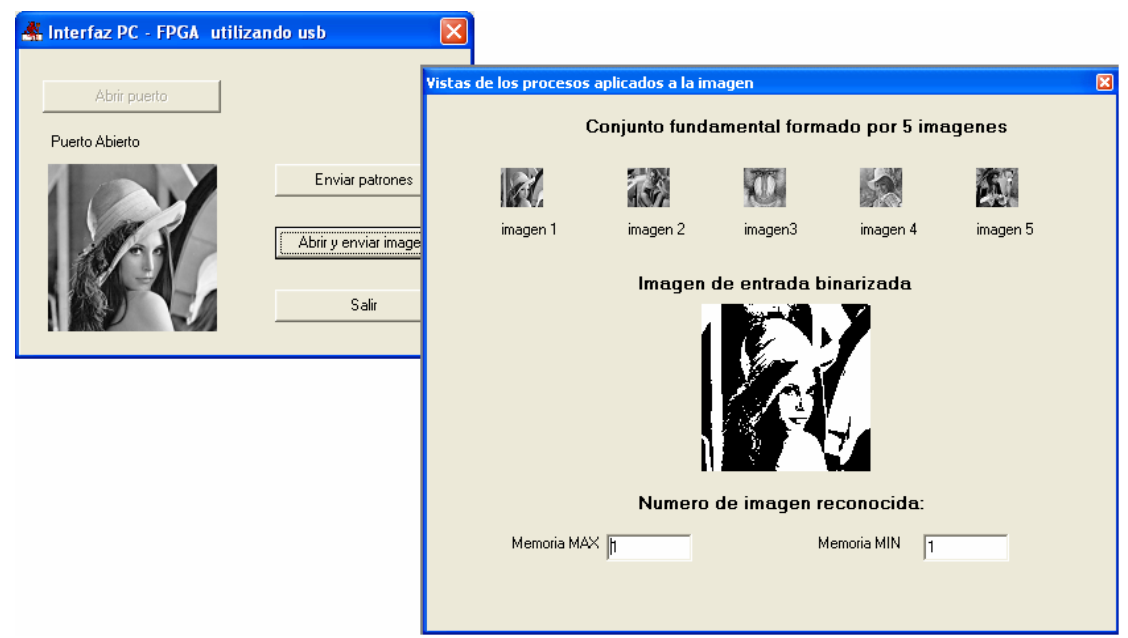

Fig. 8. USB interface between image recognition system and PC

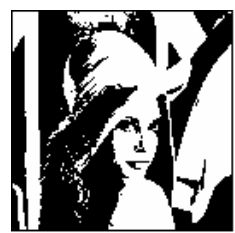

(a)

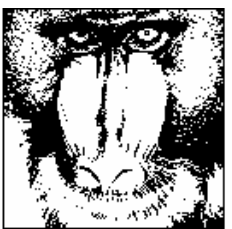

(b)

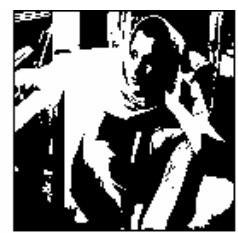

(c)

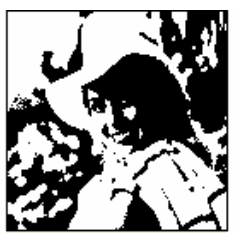

(d)

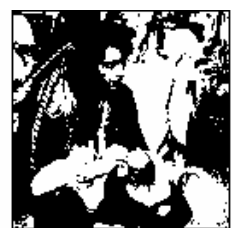

(e)

Fig. 9. Image binarization: (a) Lena, (b) Baboon, (c) Barbara, (d) Elaine, (e) Man

Table 5. Image recognition system performance on original images

\begin{tabular}{lc}
\hline Image & MHM min performance (recognition percentage) \\
\hline Lena & 100 \\
Baboon & 100 \\
Barbara & 100 \\
Elaine & 100 \\
Man & 100 \\
\hline
\end{tabular}

Table 6. Image recognition system performance on corrupted images

\begin{tabular}{lcccccc}
\hline & \multicolumn{3}{c}{ MHM min performance (recognition percentage) } \\
\cline { 2 - 7 } Image & \multicolumn{2}{c}{ Dilative Gausian noise } & \multicolumn{3}{c}{ Erosive } & Gaussian noise \\
\cline { 2 - 7 } & $10 \%$ & $20 \%$ & $30 \%$ & $10 \%$ & $20 \%$ & $30 \%$ \\
\hline Lena & 100 & 100 & 100 & 0 & 0 & 0 \\
Baboon & 100 & 100 & 100 & 0 & 0 & 0 \\
Barbra & 100 & 100 & 100 & 0 & 0 & 0 \\
Elaine & 100 & 100 & 100 & 100 & 0 & 0 \\
Man & 100 & 100 & 100 & 100 & 0 & 0 \\
\hline
\end{tabular}


Table 6. If an image is corrupted with erosive noise, it will be considered by the patterns recognition processor as an image corrupted with dilative noise, consequently the processor has a perfect recognition with images corrupted with up to $30 \%$ of dilative noise.

We used the hardware description language VHDL for the modeled system. It is a standardized language, then the design of the image recognition system is portable to any FPGA architecture. Table 7 shows FPGA design summary obtained by ISE Foundation tool.

Table 7. FPGA design summary of the image recognition system obtained by ISE Foundation tool

\begin{tabular}{lc}
\hline & Features summary \\
\hline Device & XCV300-PQ240 \\
Number of Slice & 1337 out of 3072 $(43 \%)$ \\
Number of Slice Flip Flops & 1025 out of $6144(16 \%)$ \\
Number of 4 input LUTs & 2270 out of $6144(36 \%)$ \\
Number of bonded IOBs & 64 out of $170(37 \%)$ \\
Number of TBUFs & 48 out of $3072(1 \%)$ \\
Number of GCLKs & 1 out of $4(25 \%)$ \\
Maximum Frequency & $34.254 \mathrm{MHz}$ \\
\hline
\end{tabular}

Tables 8, 9 and 10 show the processing speed of the DWT, MAM learning process and MAM recognition process. System can operate in an ample frequency range. Therefore, the speed of processing is expressed in clock cycles. The number of clock cycles for each of the considered processing stages mostly is the time consumed by access to system memory.

Table 8. Processing speed of DWT process

\begin{tabular}{lc}
\hline DWT decomposition & Number of clock cycles \\
\hline One-scale & $1,036,800$ \\
Two-scale & 259,200 \\
Three-scale & 64800 \\
Four-scale & 16,200 \\
Total & $1,377,000$ \\
\hline
\end{tabular}

Table 9. Processing speed of MAM learning process

\begin{tabular}{lc}
\hline MAM learning process & Number of clock cycles \\
\hline One image & 47,250 \\
Five images & 236,250 \\
\hline
\end{tabular}

Table 10. Processing speed of MAM recognition process

\begin{tabular}{lc}
\hline MAM recognition process & Number of clock cycles \\
\hline One image & 33,750 \\
\hline
\end{tabular}




\section{Conclusions}

The MAM have demonstrated to be an excellent tool in the recognition and recovery of patterns, due to their useful features. The obtained results confirm that MAM are robust to dilative, erosive or random noise and have great capacity to save system memory. Additionally, MAM have demonstrated high speed in both learning and recovery processes. The description of the processor for patterns recognition based on MAM using a standardized hardware description language allowed to design a system that has features as portability and easy adaptation to diverse operation circumstances. Moreover, it is possible to realize parallel processing implying high speeds of processing and high performance that grants the implementation of a hardware algorithm. The combination of all of these features resulted in a robust, fast and reliable artificial vision system that can be used in Real-Time Autonomous Systems.

\section{References}

1. Wang, L., Der, S.Z., Nasrabadi, N.M.: Automatic Target Recognition Using a FeatureDecomposition and Data-Decomposition Modular Neural Network. IEEE Transactions on Image Processing 7(8), 1113-1121 (1998)

2. Sim, D.-G, Kwon, O.-K., Park, R.-H.: Object Matching Algorithms Using Robust Hausdorff Distance Measures. IEEE Transactions on Image Processing 8(3), 425-429 (1999)

3. Young, S.S., Scott, P.D.: Object Recognition Using Multilayer Hopfield Neural Network. IEEE Transactions on Image Processing 6(3), 357-372 (1997)

4. Young, S.S., Scott, P.D., Bandera, C.: Foveal Automatic Target Recognition Using a Multiresolution Neural Network. IEEE Transations on Image Processing 7(8), 1122-1135 (1998)

5. Barcala, J.M., Alberdi, J., Navarrete, J.J., Oller, J.C.: Clasificación Automática de Envases Plásticos. XX Jornadas de Automática, Comite Español de Automática, España (1999)

6. Foltyniewicz, R.: Automatic face recognition via wavelets and mathematical morphology. In: Proceedings of the 13th International Conference on Pattern Recognition, vol. 2, pp. 13-17 (1996)

7. Hopfield, J.J.: Neural Networks and Physical Systems with Emergent Collective Computational Abilities. Proceedings of the National Academy of Sciences of the USA 79, 2554-2558 (1982)

8. Ritter, G.X., Sussner, P., Díaz de León, J.L.: Morphological Associative Memories. IEEE Transactions on Neural Networks 9(2), 281-293 (1998)

9. Yáñes, C., de León, J.L.D.: Memorias Morfológicas Heteroasociativas. Centro de Investigación en Computación, IPN, México, IT. Serie Verde 57 (2001) (ISBN 970-186697-5)

10. Yáñes, C., de León, J.L.D.: Memorias Morfológicas Autoasociativas. Centro de Investigación en Computación, IPN, México, IT 58, Serie Verde (2001) (ISBN 970-186698-3)

11. Guzman, E., Pogrebnyak, O., Yáñez, C., Moreno, J.A.: Image Compression Algorithm Based on Morphological Associative Memories. In: CIARP 2006. LNCS, vol. 4225, pp. 519-528. Springer, Heidelberg (2006) 
12. Ritter, G.X., Ritter, G.X., de León, J.L.D., Sussner, P.: Morphological Bidirectional Associative Memories. Neural Networks 12(6), 851-867 (1999)

13. Castellanos, C.: Díaz De León, J.L., Sánchez, A.: Análisis Experimental con las Memorias Asociativas Morfológicas. XXI Congreso Internacional de Ingeniería Electrónica, Electro 1999, México, pp. 11-16 (1999)

14. Pardo, F., Boluda, J.A.: VHDL: lenguaje para síntesis y modelado de circuitos, 2nd Edition. Editorial RA-MA, España (2004) (ISBN 84-7897-595-0)

15. Acharya, T., Tsai, P.-S.: JPEG2000 Standard for Image Compression. John Wiley \& Sons, Chichester (2005) 